\section{Störmerbahnen energiereicher Teilchen der kosmischen Strahlung in einer Modellgalaxis}

\section{K. O. Thielheim}

Institut für Reine und Angewandte Kernphysik, Universität Kiel

(Z. Naturforsch. 24 a, $1664-1665$ [1969]; eingegangen am 21. August 1969)

Nach den in den letzten Jahren bekannt gewordenen Ergebnissen besteht das Magnetfeld der galaktischen Scheibe aus einem großräumig geordneten und einem irregulären Anteil. Die Feldlinienbündel des ersteren sind in die galaktischen Arme eingefroren und folgen somit deren Spiralstruktur (Intensität der $21 \mathrm{~cm}$-Linie, optische Flächenphotometrie). Es ist nicht sicher bekannt, ob das magnetische Feld offen oder geschlossen ist. Die letztere Vorstellung wird durch das Speichervermögen der Galaxis für die niederenergetische kosmische Strahlung unterstützt.

Nach den Vorstellungen des quasilongitudinalen Modells verlaufen die Feldlinien im wesentlichen parallel zu den Armen mit verschiedenen Orientierungen auf beiden Seiten der ausgezeichneten Ebene. Nach dem helikalen Modell sind sie schraubenförmig, wobei die Windungen bei einem Anstellwinkel $>75^{\circ}$ um etwa $50^{\circ}$ um die kleinere Achse des elliptischen Querschnitts verschert sind. Die mittlere Feldstärke beträgt etwa $5 \cdot 10^{-6} \mathrm{G}$. $\mathrm{Zu}$ diesen Ergebnissen ${ }^{1}$ haben experimentelle Methoden (Sternpolarisation, Synchrotronstrahlung, Faraday-Drehung an extragalaktischen Quellen, Zeeman-Effekt an der $21 \mathrm{~cm}$-Linie) und indirekte Schlüsse (Struktur von Filamentnebeln, Stabilität der galaktischen Arme speziell bei differentieller Rotation, Isotropie der niederenergetischen kosmischen Strahlung) beigetragen.

Die Energiedichte der turbulenten Komponente ist wenigstens um eine Zehnerpotenz kleiner als die der großräumig geordneten Komponente. Die Abmessungen der irregulären Strukturen auf Grund verschiedener Beobachtungsverfahren (Dispersion stellarer Polarisationswinkel, Polarisation der Synchrotron-Hintergrundstrahlung, Depolarisation, Streuung des Drehungsmaßes) und indirekter Schlüsse (Größe der Wolken interstellaren Wasserstoffs) ergeben sich zu einigen $10^{-1}$ pc bis zu einigen 10 pc.

Es erscheint sinnvoll auf dieser Grundlage Betrachtungen über den Einfluß des interstellaren Magnetfeldes auf die Ausbreitung energiereicher Teilchen der kosmischen Strahlung in der galaktischen Scheibe anzustellen. Für diese Teilchen ist das Feld praktisch stationär, weil es in das interstellare Medium eingefroren ist und Bewegungen in demselben durch die Alfvén-Geschwindigkeit ( $\approx 10 \mathrm{~km} / \mathrm{sec})$ beschränkt sind. Charakteristische Längen, welche hierbei zunächst ins Spiel kommen, sind: Die Abmessungen der irregulären

Sonderdruckanforderungen erbeten an Univ.-Doz. Dr. K. O. Thielheim, Institut für Reine und Angewandte Kernphysik der Universität Kiel, D-2300 Kiel, Olshausenstr. 40/60, Gebäude 32 .
Strukturen $d_{\text {irr }} \lesssim 20 \mathrm{pc}$, der Larmor-Radius von Protonen $r_{\mathrm{L}}[\mathrm{pc}] \approx 2 \cdot 10^{-16} \mathrm{E}[\mathrm{eV}]$ und der kleinere Durchmesser eines galaktischen Armes $d_{\mathrm{A}} \approx 200 \mathrm{pc}$, ferner die wegen der Wechselwirkung mit dem Restgas endliche Lebensdauer von Protonen $l_{\text {int }}[\mathrm{kpc}] \approx 500 / \mathrm{N}$ $\left[\mathrm{cm}^{-3}\right]$. Im Energiebereich $E \gtrsim 10^{17} \mathrm{eV}$ kann die Turbulenz als Feinstruktur des Feldes angesehen werden. Bei $E \approx 10^{18} \mathrm{eV}$ ist $r_{\mathrm{L}} \approx d_{\mathrm{A}}$, so daß der galaktische Speichermechanismus versagt. Unterhalb dieses Wertes ist ein Übergangsbereich von galaktischer zu extragalaktischer Strahlung zu erwarten.

Es liegt nahe, ein Modell der großräumig geordneten Feldkomponente zu konstruieren und ihm eine durch Zufallsfunktionen des Ortes definierte, irreguläre Komponente zu überlagern, um durch Anwendung der Fokker-Planck-Gleichung die Ausbreitung der kosmischen Strahlung zu untersuchen. Eine grobe Vorstellung über den Teilchentransport kann im Bereich $E \gtrsim 10^{17} \mathrm{eV}$ unter Vernachlässigung der Feldirregularitäten durch Berechnung individueller Teilchenbahnen gewonnen werden.

$\mathrm{Zu}$ diesem $\mathrm{Zweck}$ wurde das folgende Modell entwickelt, dessen Geometrie an eine idealisierte Spiralgalaxie vom mittleren Sb-Typ erinnert. Seine Abmessungen sind denen unserer Galaxie ${ }^{2}$ angepaßt. Das Feld besitzt eine geschlossene, quasilongitudinale Struktur. Die mittlere Feldstärke beträgt in den Armen $\approx 5 \cdot 10^{-6} \mathrm{G}$. Andererseits wird das Feld im zentralen Bereich nicht beschrieben, weil dessen Abmessungen verhältnismäßig klein sind. Desgleichen wird das Halo. feld nicht beschrieben, weil dessen Existenz umstritten ist. Es wird nicht versucht, Einzelheiten der lokalen Feldstruktur wiederzugeben.

Das Modell wird in Zylinderkoordinaten $R, z, \varphi$ formuliert. In der Ebene $z=0$ wird die Spirale

$$
\varphi(R)=a(R / b) \operatorname{arctg}(R / b)+\varphi_{0}
$$

mit den Parametern $a=1, b=1,5 \mathrm{kpc}$ und $\varphi_{0}=0$ definiert. Durch Rotation um die $z$-Achse und Verschiebung parallel zur $z$-Achse kann aus den Tangentenvektoren an die Spirale in jedem Punkt ein Einheitsvektor $a_{0}$ gewonnen werden, auf den die zur $z$-Achse senkrechte Komponente

$$
\begin{aligned}
H_{a_{0}}= & c z \exp \left\{-z^{2} / z_{0}^{2}-R^{2} / R_{0}^{2}\right\} \cdot\left(1-\exp \left\{-R^{2} / R_{1}^{2}\right\}\right) \\
& \cdot\left(1+k^{2} \cos ^{2}[\varphi-\varphi(R)]\right)
\end{aligned}
$$

bezogen ist. Die Parameter sind $c=5 \cdot 10^{-5} \mathrm{G} / \mathrm{kpc}$, $z_{0}=0,175 \mathrm{kpc}, k=2, R_{0}=10 \mathrm{kpc}, R_{1}=2 \mathrm{kpc}$. Die zur $z$-Achse parallele Feldkomponente

$$
\begin{aligned}
H_{z_{0}}= & c z_{0}^{2} \exp \left\{-z^{2} / z_{0}^{2}-R^{2} / R_{0}^{2}\right\} \cdot\left(1+t^{2}\right)^{-1 / 2} \\
& \cdot\left(1+k^{2} \cos ^{2}[\varphi-\varphi(R)]\right) \\
& \cdot\left(( 1 - \operatorname { e x p } \{ - R ^ { 2 } / R _ { 1 } ^ { 2 } \} ) \cdot R ^ { - 1 } \cdot \left[\left(1+t^{2}\right)^{-1}\right.\right. \\
& \left.\cdot\left[1-2 a b^{2} R^{2} t\left(b^{2}+R^{2}\right)^{-2}\right]-2 R^{2} / R_{0}^{2}\right] \\
& \left.+2 R \exp \left\{-R^{2} / R_{1}^{2}\right\} / R_{1}^{2}\right) \\
& t=\varphi(R)+a R^{2} /\left(b^{2}+R^{2}\right)
\end{aligned}
$$

1 H. C. v. D. Hulst, Ann. Rev. Astr. Astrophys. 8, 167 [1967]; u. v. a.

2 A. Unsöld, Der neue Kosmos, Springer-Verlag, Berlin 1967. 
folgt aus $\operatorname{div} H=0$ und geeigneten Randbedingungen. Die Bewegungsgleichungen

$r^{\prime \prime}=\alpha\left[r^{\prime}, H\right]$ mit $\alpha\left[\mathrm{G}^{-1} \mathrm{kpc}^{-1}\right]=0,925 \cdot 10^{24} E^{-1}[\mathrm{eV}]$ werden in ein System von Differentialgleichungen erster Ordnung übergeführt und numerisch integriert. Für jeden betrachteten Wert der Energie werden formal Bahnen von negativ geladenen Teilchen berechnet, die unter verschiedenen Richtungen vom Ort der Erde ausgehen.

Vorläufige Rechnungen ${ }^{3}$ haben gezeigt: Oberhalb von $10^{19} \mathrm{eV}$ werden die Bahnen der Protonen vom Modellfeld praktisch nicht beeinflußt. Bei $10^{18} \mathrm{eV}$ besitzt das Feld fokussierende Eigenschaften, Teilchen, welche die Erde erreichen, kommen aus der Umgebung

3 K. O. Thielheim u. W. Langhoff, Nature 219, 355 [1968]; Proc. Phys. Soc. London (Ser. 2) 1, 351 [1968]. des Antizentrums. Aus der Umgebung des Zentrums gelangen keine Teilchen zur Erde. Bei $10^{17} \mathrm{eV}$ und darunter haben die Gyrationszentren die Tendenz, den Feldlinien zu folgen. Teilchen aus dem Metagalaktischen Bereich legen in den galaktischen Armen lange Wege zurück, bevor sie die Erde ererichen, der Speichermechanismus wird wirksam. Unterhalb von $2 \cdot 10^{16}$ $\mathrm{eV}$ ist dieser Weg für alle Richtungen der einfallenden Teilchen größer als $100 \mathrm{kpc}$, oberhalb von $6 \cdot 10^{17} \mathrm{eV}$ kleiner als $100 \mathrm{kpc}$. Diese Werte sollten grob den Grenzen des Übergangsbereiches entsprechen. Sie können daher mit den Knicks des primären Energiespektrums in Zusammenhang gebracht werden.

Ich danke der Deutschen Forschungsgemeinschaft für die unter dem Zeichen Th 104/4 gewährte finanzielle Unterstützung der Arbeit.

\section{Zur Präparation laseraktiver Flüssigkeiten mit hohen Fluoreszenzlebensdauern}

\section{H. WeichSelgartNer}

Institut für Plasmaphysik, Garching bei München

(Z. Naturforsch. 24 a, 1665-1666 [1969] ; eingegangen am 7. August 1969)

Im Herbst 1966 berichteten Heller und Lempicki ${ }^{1}$ über einen bei Zimmertemperatur arbeitenden Neodymflüssigkeitslaser mit Selenoxychlorid als Lösungsmittel. Wir haben die Arbeiten an diesem Lasertyp ebenfalls begonnen und kommen bei einer etwas anderen Präparationstechnik als HELLER ${ }^{2}$ zu ähnlichen Ergebnissen. Über die physikalischen Ergebnisse, die mit stationären und Kreislauf-Lasern erzielt wurden, wird von LANG $^{3}$ an anderer Stelle berichtet werden.

Hier soll nun ein kleines Gerät beschrieben werden, mit dem auf einfachste Weise die äußerst feuchtigkeitsempfindlichen Laserflüssigkeiten hergestellt und in Küvetten überführt werden können.

Ein $50-100 \mathrm{ml}-$ Kolben trägt einen angeschmolzenen Rückflußkühler und kann durch eine NS 14.5-Hülse beschickt werden. Oberhalb des Kühlers sitzen 2 Fritten mit 14 bzw. 1,1 $\mu$ mittlerer Porenweite

Der Filtratablauf wird durch einen NS 14,5-Kern geführt, so daß das Filtrat den Schliff nicht benetzen kann. Ein Pumpenhahn V führt zu einer mit 2 Kühlfallen (fl. $\mathrm{N}_{2}$-befüllt) geschützten Vorvakuumpumpe (RZ 12). Die Küvette hat 2 Anschlüsse, deren erster mit dem Schliffkern des Gerätes verbunden ist; durch den zweiten wird die Küvette nach Beschickung mit der fertigen Lösung mit nachgereinigtem $\mathrm{N}_{2}$ oder $\mathrm{He}$ belüftet und anschließend verschlossen.

$\mathrm{Da}$ die verwendeten Lösungsmittel (in erster Linie $\mathrm{SeOCl}_{2}$ ) sämtliche Schliffette angreifen, werden die Schliffe mit ungesintertem Teflonband gedichtet. Das Ventil V kann mit Kel F 90 gefettet werden.

Sonderdruckanforderungen erbeten an Dr. H. WEICHSELGARTNER, Institut für Plasmaphysik GmbH, D-8046 Garching $b$. München.

1 A. Lempicki u. A. Heller, Appl. Phys. Lett. 9, 108 [1966].

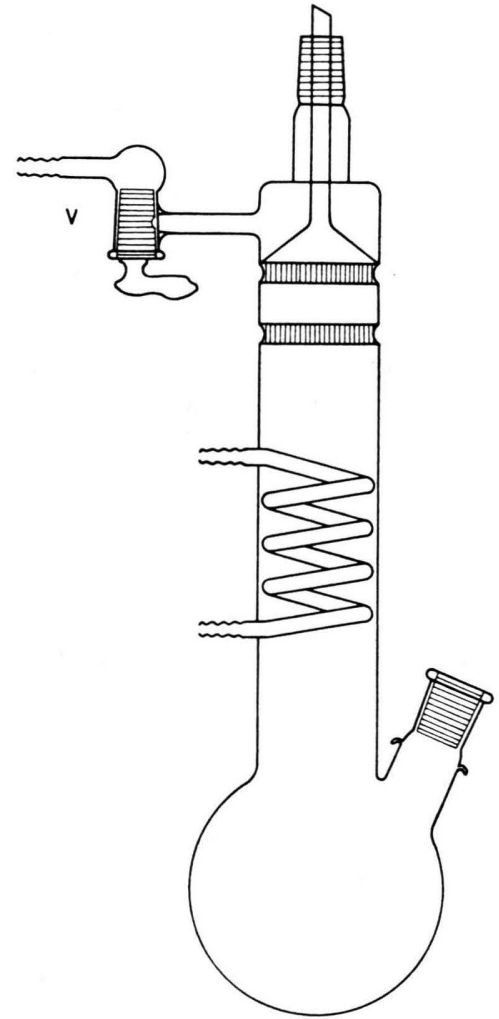

Abb. 1. Apparatur ELF zur Präparation und Abfüllung laseraktiver Lösungen.

Zur Präparation werden $3 \mathrm{~g} \mathrm{Nd}_{2} \mathrm{O}_{3}$ zusammen mit $50 \mathrm{ml} \mathrm{SeOCl}{ }_{2}$ und $10 \mathrm{ml} \mathrm{SbCl}{ }_{5}$ unter Schutzgas-Spülung in den Kolben gegeben und bei langsam gestei-

2 A. Heller, J. Am. Chem. Soc. 90 (4), (3. 7.68), 3711 [1968].

${ }^{3}$ R. LANG, IPP. 